\title{
Genetics of Cleft Palate and Velopharyngeal Insufficiency
}

\author{
Walter M. Sweeney ${ }^{1} \quad$ Steve T. Lanier ${ }^{1} \quad$ Chad A. Purnell $^{1} \quad$ Arun K. Gosain $^{1}$ \\ ${ }^{1}$ Division of Plastic Surgery, Ann and Robert H. Lurie Children's \\ Hospital of Northwestern University, Chicago, Illinois, United States \\ J Pediatr Genet 2015;4:9-16.

\begin{abstract}
Address for correspondence Arun K. Gosain, MD, Division of Pediatric Plastic Surgery, Box 93, Lurie Children's Hospital of Northwestern University, Feinberg School of Medicine, $225 \mathrm{E}$. Chicago Ave., Box 93, Chicago, IL 60611, United States
\end{abstract} \\ (e-mail: argosain@luriechildrens.org).
}
Abstract
Keywords
- cleft palate
- velopharyngeal insufficiency
- palate anatomy
- palate physiology
- velopharynx
- genetics

Velopharyngeal insufficiency (VPI) can occur in the setting of an unrepaired or repaired cleft lip and palate. The rate of VPI has been documented as high as 33\% in some studies with higher rates of recurrences following surgery associated with genetic syndromes such as $22 q 11.2$ deletions. The primary cause of VPI in these groups is still identified as the anatomic abnormalities of the velum. In this review, the anatomy and physiology of the velum are discussed along with genetic mutations associated with VPI.

\section{Introduction}

Clefting of the lip and/or palate is an embryological phenomenon that reflects a failure of fusion during maxillary and palatal development. Failure of fusion of the medial nasal processes and maxillary processes can result in clefting of the lip, alveolus, and primary palate. During palatal development, the lateral palatal shelves typically fuse in the 7th to 8th week of gestation by rotating from a vertical to horizontal orientation. Fusion of the palate proceeds from the anterior to posterior direction. ${ }^{1}$ The failure of fusion results in a spectrum of esthetic and functional deformities, considered among the most critical is the potential disruption of speech development. Meeting appropriate speech milestones is dependent on the functional and structural integrity of the velopharynx. The velopharynx is a complicated integrated structure that separates the oral and nasal cavities during speech production. Velopharyngeal dysfunction can result in numerous speech abnormalities including compensatory articulation, hypernasality, and nasal air emissions, which can impair intelligibility. ${ }^{2}$ The goals of cleft palate repair are to reestablish competence of the velopharyngeal mechanism, so

received

October 12, 2014

accepted after revision

December 26, 2014

as to enable and prevent nasal regurgitation of air and fluids. ${ }^{3}$ Accomplishing these goals and optimizing outcomes require an in-depth understanding of anatomy and velopharyngeal dynamics.

\section{Historical Perspective}

In 1865, Passavant ${ }^{4}$ attempted the first recorded correction of the velopharynx with adhesion of the soft palate to the posterior pharynx. This was followed by the pharyngeal flap in 1875, which was introduced by Sloan, ${ }^{5}$ and in 1930 , the pharyngeal flap was first described in the United States by Padgett. ${ }^{6}$ The pharyngeal flap has been a cornerstone in the management of velopharyngeal insufficiency (VPI) but has been associated with increased rates of obstructive sleep apnea secondary to inadequately sized lateral ports. ${ }^{5}$ Because of the aforementioned complications, several modifications to the pharyngeal flap have been proposed and the role of the pharyngeal flap in management of VPI is continually being reassessed.

Velopharyngeal incompetence was then addressed by Wilfred Hynes ${ }^{7,8}$ in 1950, when he proposed utilizing

Copyright $\odot 2015$ by Georg Thieme Verlag KG, Stuttgart · New York
DOI http://dx.doi.org/ 10.1055/s-0035-1554978. ISSN 2146-4596. 
myomucosal flaps composed of salpingopharyngeus and palatopharyngeus muscles, which would be sutured to the posterior pharyngeal wall. This technique has undergone multiple modifications, starting with Jackson et $\mathrm{al}^{9}$ in 1968 who proposed including an inferiorly based myomucosal flap. In 1977, Jackson and Silverton ${ }^{10}$ endorsed integration of palatopharyngeal flaps and a superiorly based pharyngeal flap. Riski et $\mathrm{al}^{11}$ modified this approach by promoting suturing the myomucosal flaps further cephalad on the posterior pharynx where the velum makes contact. Alterations in the above techniques continue to be documented and published.

Augmentation of the posterior pharyngeal wall is a technique that has been proposed to decrease the size of the velopharyngeal gap and restore competence. Vaseline was recorded being used in 1900 along with several other materials including, but not limited to, porous polyethylene, Proplast, collagen, calcium hydroxyapatite, and autologous tissue. ${ }^{12,13}$ Posterior pharyngeal wall augmentation has several disadvantages, many of which are directly related to the type of material chosen for augmentation.

\section{Velopharyngeal Anatomy and Physiology}

The borders of the velopharynx, also referred to as the velopharyngeal port, include the soft palate anteriorly, posterior pharyngeal wall posteriorly, and the lateral pharyngeal walls laterally. Phonation requires closure of the velopharyngeal port, which is initiated by higher motor cortical functions resulting in coordinated contractions of the velopharyngeal musculature. The muscles of the soft palate (also referred to as the velum) include the tensor veli palatini, palatoglossus, palatopharyngeus, levator veli palatini, and musculus uvulae. ${ }^{14,15}$

\section{Levator Veli Palatini}

The levator veli palatini is critical for closure of the velopharyngeal port. The muscle originates from the petrous portion of the temporal bone and the junction of the bony and cartilaginous Eustachian tube. The levator veli palatine muscle fibers course anteriorly, medial, and inferiorly to insert into the middle of the velum, or more specifically, into the palatal aponeurosis interdigitating in a horizontal fashion with muscles from the contralateral side. The palatal aponeurosis is a thin fibrous structure that provides structural integrity to the velum and is a junction between the hard and soft palate. The decussation of the paired levator fibers in the palatal aponeurosis creates a muscular sling, which when contracted will retract and elevate the velum at a 45-degree angle against the posterior pharyngeal wall. This is the primary mechanism of velopharyngeal port closure. ${ }^{16,17}$

\section{Musculus Uvulae}

The musculus uvulae is a paired intrinsic muscle that rests within the levator veli palatini muscular sling. The term intrinsic refers to the fact that the musculus uvulae has no external attachments and resides completely within the velum. The muscle fibers of the musculus uvulae fibers originate from the palatal aponeurosis and course posteriorly along the nasal surface of the velum until it reaches the uvulae. The musculus uvulae provides additional tissue bulk on the dorsal surface of the velum that assists in closure of the velopharyngeal port.

\section{Tensor Veli Palatini}

The tensor veli palatini is a broad thin muscle that originates from the scaphoid fossa at the base of the medial pterygoid plate of the sphenoid bone and lateral cartilaginous wall of the eustachian tube. The muscle then travels vertically between the medial pterygoid plate and medial pterygoid muscle to become the tensor tendon, which travels around the hamulus of the medial pterygoid plate before inserting into the palatal aponeurosis immediately posterior to the posterior nasal spine. ${ }^{18,19}$ The tensor veli palatini will primarily open the eustachian tube during swallowing and yawning, permitting drainage of fluids in the middle ear and equalizing pressure. In patients with cleft palate, the abnormal insertion and function of the tensor is thought to be the source of frequent otitis media. $^{20}$

\section{Superior Pharyngeal Constrictor}

The superior pharyngeal constrictor is located in the pharynx and is one of three pharyngeal constrictors (superior, middle, and inferior pharyngeal constrictors). Of these muscles, the superior pharyngeal constrictor is located in the pharynx. The appearance of the superior constrictor can distinguish it from the others within the pharynx, as it is thinner and paler in appearance. The superior constrictor is divided into the following four parts: (1) pterygopharyngeal, (2) buccopharyngeal, (3) mylopharyngeal, and (4) glossopharyngeal. These muscle bundles have several different origins including the medial pterygoid plate, pterygomandibular raphe, and alveolar process. These muscles fibers will contribute to the lateral and posterior pharyngeal walls and converge in the midline to form the midline raphe. Some fibers will orient horizontally giving rise to the Passavant ridge, and other fibers will insert onto the velum and assist in contraction and closure of the velopharyngeal port. $^{21}$

\section{Palatopharyngeus}

The palatopharyngeus is a vertically oriented muscle located in the posterior tonsillar pillar, with a transverse muscular component referred to as the palatopharyngeus proper. The palatopharyngeus originates from the velum's soft palate and courses posteriorly to insert on the lateral pharyngeal walls and greater horns of the thyroid cartilage. The transversely oriented fibers cause anterior inward displacement of the lateral pharyngeal walls and contribute to the Passavant ridge. $^{22}$ Vertically oriented fibers assist with positioning of the velum. The palatopharyngeus' main function will be to contract over a bolus of food and prevent migration into the nasopharynx. The palatopharyngeus works in complement to the levator palatini to achieve velopharyngeal closure, with the transverse fibers pulling the lateral pharyngeal 
walls medially during closure of the velopharyngeal port. $22-24$

\section{Palatoglossus}

The palatoglossus muscle courses from the lateral margins of the velum, through the anterior tonsillar pillar, and inserts onto the lateral aspect of the tongue. The palatoglossus acts as a direct antagonist to the levator veli palatini and contraction of the palatoglossus lowers the velum. Muscle fibers within the anterior tonsillar pillar will provide passive restoration on the velum to help lower the palate and open the velopharyngeal port to assist with speech production. ${ }^{25}$ The palatoglossus also elevates the posterior tongue, which plays a role in swallowing and manipulating a food bolus in an anterior and posterior direction toward the esophagus. Interdigitation of palatal muscles, including the palatoglossus and palatopharyngeus, work in concert to provide velar positioning in adjunct to the primary elevating function of the levi veli palatini to elevate the velum.

\section{Salpingopharyngeus}

The salpingopharyngeus originates at the torus tubaris adjacent to the superior border of the medial cartilage of the eustachian tube. ${ }^{26}$ The muscles fibers then pass inferiorly in the salpingopharyngeal fold to intertwine with the palatopharyngeus muscle of the lateral pharyngeal walls. The presence and size of this muscle is variable between individuals and not known to have any critical function to closing the velopharyngeal port or in the positioning of the velum. ${ }^{27}$

\section{Motor Innervation of the Velum}

Motor innervation of the velum is supplied primarily by the vagus nerve through the pharyngeal plexus along with the mandibular division of the trigeminal nerve. The vagus nerve primarily innervates the entire velum, including the critical function of the levator veli palatini. The vagus nerve also innervates the external muscles of the pharynx, which include the superior constrictor, middle constrictor, and inferior constrictor. The mandibular division of the trigeminal nerve innervates the tensor veli palatini, which as previously mentioned functions to open the auditory tube.

\section{Important Functional Anatomy of the Cleft Palate}

Clefting displays a spectrum of abnormalities, which impact the degree of functional deficits, surgical approach, and timing of surgical repair (i.e., submucosal cleft palate). However, to have an understanding of VPI, an understanding of how clefting affects velar anatomy is essential. The abnormal position of the levator veli palatini is critical to the functional deficits observed in cleft palate patients. As mentioned previously, the levator veli palatini forms a transverse sling across the posterior portion of the velum and during contraction moves the velum superiorly and posteriorly. This action places the velum in contact with the posterior pharyngeal wall at the level of the adenoid pad, which will close the velopharyngeal port. In cleft palate, the levator veli palatini is

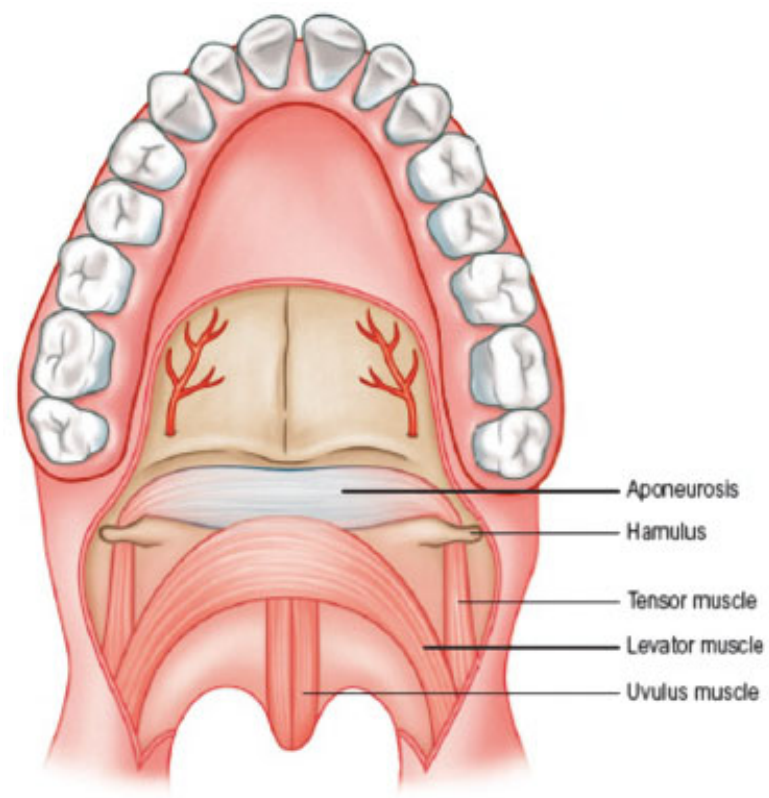

Fig. 1 Normal palate anatomy that demonstrates the levator veli palatini muscle forming a sling, which is critical to elevating the velum.

discontinuous across the palate and runs longitudinally to insert abnormally onto the posterior aspect of the hard palate. Contraction of the levator in this position cannot reposition the velum against the posterior pharyngeal wall to close the velopharyngeal port resulting in nasal air emissions during speech formation, which manifests as the characteristic hypernasal speech observed in cleft palate patients ( - Figs. 1 and 2 ).

The levator and tensor veli palatini share a common tendinous insertion at the hamulus. This is referred to as

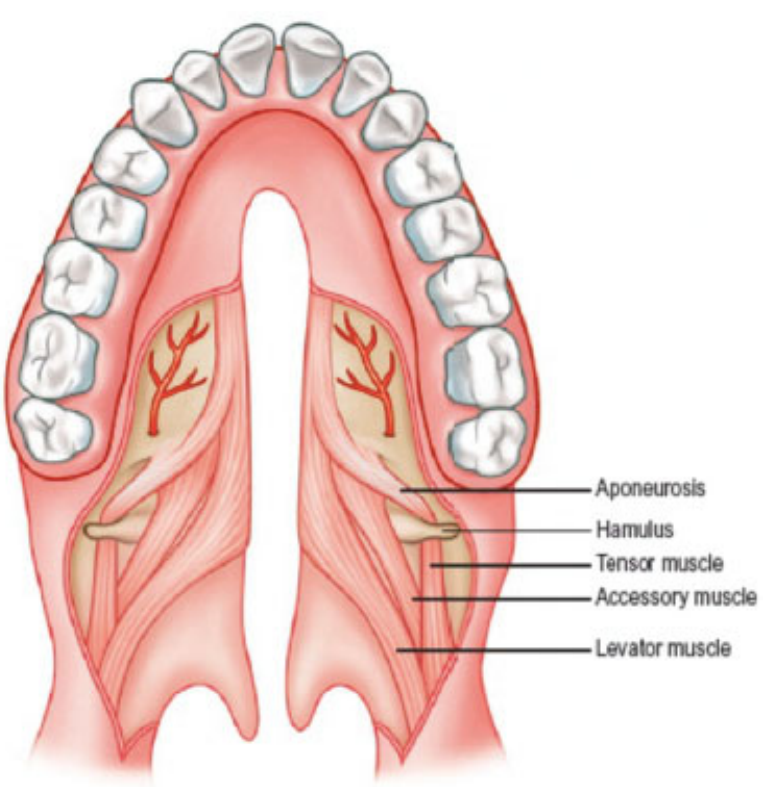

Fig. 2 Cleft palate anatomy demonstrating the levator veli palatini running in a longitudinal direction and inserting on the posterior hard palate. 
the "common tensor tendon" or the "tensor aponeurosis," and through a pulley effect it opens the Eustachian tube. In the patient with cleft palate, the abnormal anatomical position of the levator prevents it from assisting in this pulley affect. This leads to impaired opening of the eustachian tube resulting in chronic obstruction of drainage causing otitis media. It has been documented that 10 to $30 \%$ of patients with cleft palate suffer from hearing loss, which is mostly contributed to by the mechanism described earlier. ${ }^{1}$

\section{Velopharyngeal Physiology}

The velum, as discussed earlier, functions to separate the nasopharynx from the oropharynx by closing the velopharyngeal port. This is precipitated by the contraction of levator veli palatine, which elevates the velum superiorly and posteriorly against the posterior pharyngeal wall. Positioning of the velum is secondarily influenced by the palatoglossus and palatopharyngeus muscles to help for intricate adjustments of the velum. The velopharyngeal port closes approximately at the level of the palate, but the tightness of the port is influenced by the type of speech production. Contribution of the lateral pharyngeal walls to speech production varies between individuals and for the production of different sounds.

The normal anatomic closure of the velopharyngeal port can be described in the following three ways: (1) Circular, where there is equal contribution of the pharyngeal walls and velum to port closure, (2) Coronal, where closure of the port is predominated by anterior-posterior movement of the velum to the posterior pharyngeal wall, (3) Sagittal, where closure of the port is predominated by lateral pharyngeal wall movement toward the midline (-Fig. 3). However, the mechanism of velar positioning during phoneme-specific sounds is a
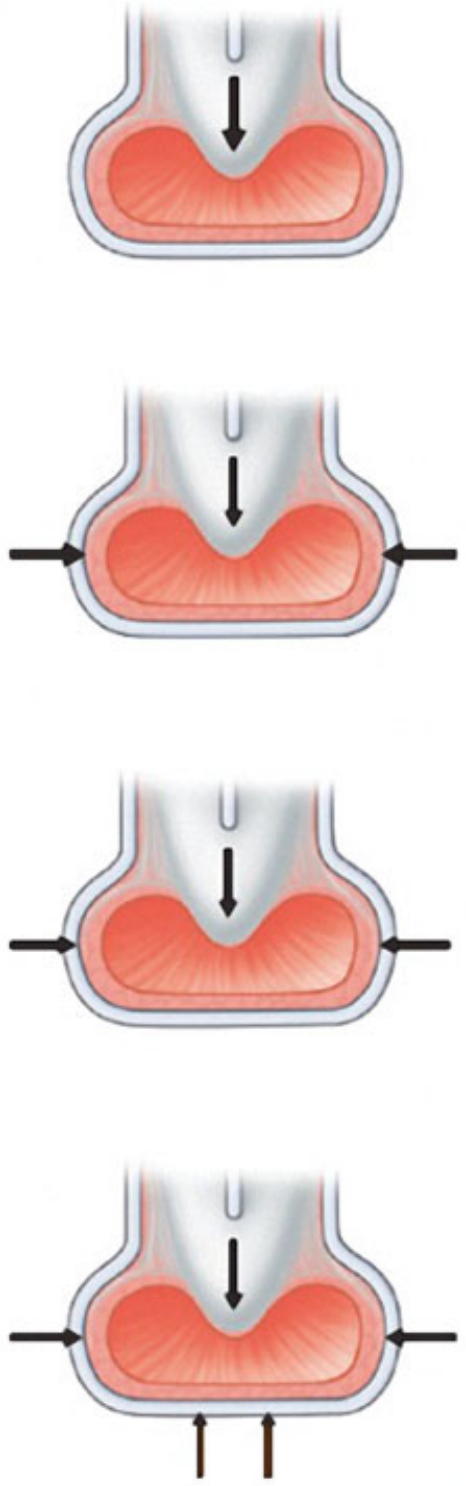

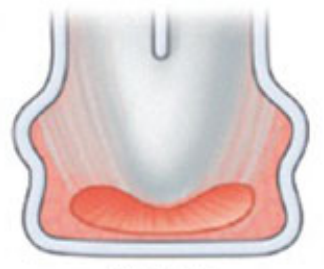

Coronal

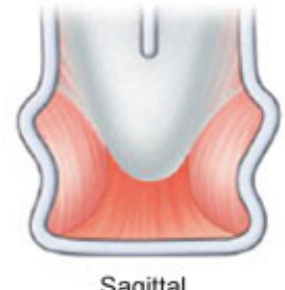

Sagittal

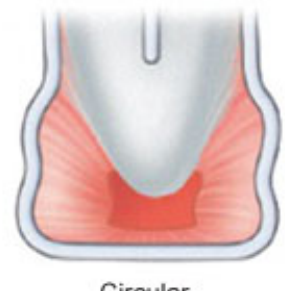

Circular
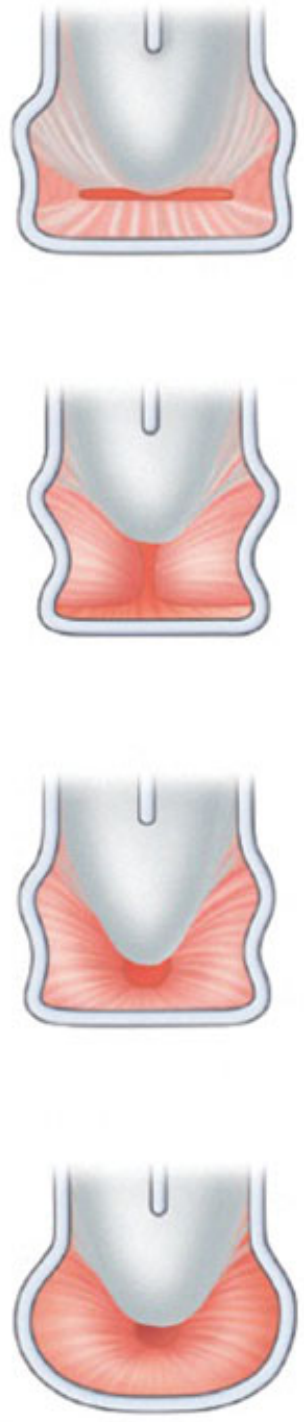

Circular with the Passavant ridge

Fig. 3 Common velopharyngeal closure patterns. 
complex interplay that extends beyond velopharyngeal port closure and this has yet to be fully elucidated. ${ }^{1}$

\section{Velopharyngeal Insufficiency and Velopharyngeal Incompetence}

Velopharyngeal dysfunction is a broad term, which describes nasal air emission during speech production and is further separated into VPI and velopharyngeal incompetence. VPI is an anatomic defect or structural abnormality, which prevents complete closure of the velopharyngeal port. The most common causes of VPI are unrepaired submucosal cleft palate or overt cleft palate. VPI can persist following palate correction secondary to a persistently dysfunctional velum, short velum, or an oronasal fistula. ${ }^{28}$ In addition, removal of enlarged adenoids can result in a velar-adenoidal VPI secondary to an enlarged pharyngeal diameter. However, in the majority of patients following adenoid removal, the velum will compensate, and closure of the port returns to normal within 12 months without intervention.

VPI refers to the inability to achieve velopharyngeal port closure secondary to a congenital or acquired neurological process (traumatic brain injury, cerebrovascular brain accident, and congenital hypotonia). Diagnostic evaluation of velopharyngeal incompetence should not demonstrate any underlying anatomical or structural abnormalities, although speech abnormalities typically parallel those found in VPI.

\section{Genetic Associations of Velopharyngeal Incompetence}

\section{Chromosome 22q11.2 Deletion Syndromes}

Approximately 1 in 4,000 children are born with 22q11.2 deletions with the majority of those children identified as DiGeorge or velocardiofacial syndrome (VCFS). 22q11.2 deletions result in a heterogeneous group of disorders that can include other disorders such as Caylor cardiofacial syndrome, corotruncal anomaly face syndrome, and Sedlackova syndrome. ${ }^{29}$ VCFS is clinically identified by cleft palate, abnormal development of thymus, parathyroids, and conotruncal defects. Approximately $75 \%$ of patients affected with VCFS have cardiac abnormalities, with the most common defect being an interrupted aortic arch. This classification is further complicated by observed heterogeneity in clinical manifestations and chromosomal deletions. 22q11.2 deletion syndromes are most commonly related to a three million base pair deletion that is flanked by a low copy number repeat. ${ }^{30}$ The deletion is a result of nonallelic meiotic recombination during either spermatogenesis or oogenesis. Predominant manifestations of 22q11.2 deletion include congenital heart disease ( $74 \%$ of individuals), palatal abnormalities (69\%), immune deficiency (77\%), and some degree of facial dysmorphology, which is present in almost every individual. As mentioned, $69 \%$ of patients with a 22q11.2 deletion have a palatal abnormality. The palatal abnormalities include cleft palate $(11 \%)$, submucousal cleft palate ( $16 \%$ of individuals), bifid uvula (5\%), and cleft lip/cleft lip and palate (2\%). Each of the aforementioned abnormalities can result in, or be asso- ciated with, VPI, which affects $27 \%$ of individuals with 22q11.2 deletions. ${ }^{28}$

VPI in 22q11.2 deletions is also related to muscle hypotonic, adenoid hypoplasia, platybasia, upper airway asymmetry, and neuroanatomical abnormalities. Muscle hypotonia was discussed in Witt et al, ${ }^{30}$ where patients were found to have poorly functioning velopharyngeal port closure. In follow-up studies, the thickness of superior pharyngeal constrictor between patients with 22q11.2 and normal controls were evaluated. The results indicated that patients with 22q11.2 deletion had considerably thinner superior constrictors and fewer type II fibers. ${ }^{31}$

During closure of the port, the velum contacts at level of the velum in children younger than the age of 5 years. ${ }^{32}$ It has been shown that hyoplastic adenoids are related to a disruption in speech patterns. Platybasia is a congenital spinal deformity defined by an increased angle between the occipital bone and cervical spine. The increased basal skull angle and flattened skull has a secondary effect of increasing the size of the velopharyngeal gap, which can worsen VPI. ${ }^{33}$ Upper airway asymmetry has also been established in patients with 22q11.2 deletion in the form of asymmetric palate elevation and incomplete velopharyngeal closure. In Chegar et $\mathrm{al}^{33}$ it was shown that $69 \%$ of patients with deletion showed the aforementioned asymmetries as compared with $20 \%$ of controls. ${ }^{34}$ Studies have also shown central nervous system abnormalities involved in thinning of the cortex in the frontal gyrus, an area critical to language development, ${ }^{35,36}$ which suggests that the etiology of abnormal speech development may extend beyond the anatomy of the velopharynx.

\section{Candidate Genes for Velopharyngeal Insufficiency}

The most common deletion present in $85 \%$ of individuals is Tbox-1 (TBX1) with the remaining individuals possessing nested deletions. However, patients who do not exhibit the classic 22q11.2 deletion phenotype have been noted to carry duplication within the 22q11.2 region that is normally deleted. ${ }^{37}$ The majority of studies examining 22q11.2 deletions have been performed in the mouse model, with more recent inquiries focused on targeted mutagenesis for the transcription factor encoding TBX1. Single gene targeting experiments in murine studies have shown several structural abnormalities including persistent truncus arteriosus, abnormalities of the pharynx, microtia, and other craniofacial malformations. ${ }^{38,39}$ Murine models have demonstrated several potential pathways for TBX1's involvement in craniofacial development. Through its role in cephalic mesoderm, TBX1 triggers the formation of muscles involved in facial expression, pharyngeal functions, and palatal formation. It is more complex than it appears on the surface, as the gene products also interact with a group of mesodermal cardiac progenitor cells within the pharyngeal region that is contiguous with the craniofacial mesoderm; these cells are referred to as second heart field cells. It is recognized that $T b x 1$ is not the only gene that interacts with second heart field cells for craniofacial morphogenesis. ${ }^{40,41}$ Coexpression of TBX1, ISL1 transcription factor (Isl1), and transcription factor 21 (Tcf21) are markers 
present on branchial myogenic progenitor cells contributing to the pharyngeal mesoderm. This expression signature is unique as compared with other myogenic precursors. However, branchial myogenic progenitor cells that carry null alleles of TBX1 have been documented to retain the ability to activate branchiomere-specific genes myogenic differentiation 1 (Myod 1) and which results in formation of hypoplastic pharyngeal muscle..$^{42}$ In addition to $T b x 1$, sine oculis-related homeobox 1 (Six1) and eyes absent 1 homolog (Eya1) have been implicated in craniofacial development through TBX1 interactions. The expression of Six1/Eya is decreased in Tbx1 nulls, suggesting that these genes are downstream from $T b x 1$, Six1 and Eya1 to form a complex that reacts as a modifier of fibroblast growth factor 8 ( $F g f 8)$; and haploinsufficiency of Fgf8 results in aberrant craniofacial and velopharyngeal formation. It is proposed that $F g f 8$ is regulated through the Six1/Eya1 complex. ${ }^{43}$

Bone morphogenetic protein (BMP) signaling plays a role in craniofacial development; and one of the proposed pathways occurs through chordin. Chordin is a BMP antagonist secreted from mesoderm that assists in determining the stereotypical dorsal-ventral tissue differentiation in vertebrates with chordin mutants developing craniofacial anomalies. Chordin is also recognized as a modifier of TBX1. Murine models have shown that chordin mutants are synergistically worsened in the presence of splice site mutations of TBX1. ${ }^{44}$ Modeling of proposed mechanisms explains this synergistic relationship through increased BMP signaling. This occurs for the following two reasons: (1) Direct loss of chordin's antagonistic effect on BMP and (2) TBX1 nulls are unable to negatively regulate SMAD family number 1 (SMAD 1) through protein interactions, and therefore SMAD acts as a BMP agonist. ${ }^{45}$ Murine models of cleft palate can be rescued with the addition of $T b x 1$, and neural crest migration in dorsal rostral patterning improved, but not corrected completely.

Interferon regulatory factor (IRF) 6 is a gene that has been linked to cleft palate formation and is responsible for Van Der Woude syndrome (VWS). VWS is an autosomal dominant condition mapped to chromosome 1q and accounts for $2 \%$ of all cleft lip and palate cases. ${ }^{46}$ The syndrome presents with congenital pits/sinuses of the lower lip in the presence of cleft lip and palate. IRF6 has a DNA-binding domain and proteinbinding domain referred to as SMAD-interferon regulatory factor-binding domain and the IRF protein typically regulates interferon following a viral infection. The gene has been shown to be highly expressed in the medial edge epithelia during palatal development and fusion within murine models around embryonic day 15 . Several studies have demonstrated IRF6's relationship with VWS and popliteal pterygium syndrome, and to date more than 200 mutations have been reported. ${ }^{47,48}$ The majority of these mutations described were either missense, nonsense, or frameshift mutations, but nucleotide substitutions have been reported.

Msh homeobox 1 (MSX1) gene is a part of the homeobox gene family that has transcripts that are represented in abundance in embryonic tissues. MSX1 has been identified as a candidate gene with significant linkage to cleft palate and oligodonita. ${ }^{49}$ The primary function of MSX1 in the develop- ment of cleft palate, stems from its primary epithelial-mesenchymal interactions, through signaling molecules. ${ }^{47,50}$ Null MSX1 murine models showed craniofacial abnormalities, cleft of the secondary palate, absence of areolar process, and oligodontia. During formation of the palate in MSX1-deficient mice the palatal shelves form and elevate normally but do note fuse. The exact mechanism of the phenomenon is not known. Recent studies have shown defective cell proliferation in the anterior palate of MSX1 mutant mice in the setting of down-regulated bone morphogenetic proteins. ${ }^{51}$ In the wildtype Bmp4 induced MSX1 supporting, the possibility of a feedback loop. Bmp4 interaction with MSX1 is also involved in expression of sonic hedgehog in medial edge epithelia. This complex interaction results in cell proliferation necessary for palatal growth.

Poliovirus receptor-related 1 (PVRL1) gene located on chromosome 11q23.3 encodes for adhesion proteins that contribute to the function of tight junctions and adherens junction in epithelial and endothelial cells. The PVRL1 protein belongs to the immunoglobulin superfamily and is calcium independent cell-cell adhesion molecule and is expressed in developing palatal epithelium. ${ }^{52}$ Mutations in this gene results in ectodermal dysplasia, referred to as Margarita Island type because of its increased frequency on an island of the same name immediately north of Venezuela. ${ }^{53}$ This is an autosomal recessive disorder characterized by cleft lip/palate, ectodermal dysplasia, and partial syndactyly of fingers and/or toes. The mutation is typically a nonsense mutation, and the Margarita Island population carries an increased rate of heterozygous mutations. It is thought that perhaps this mutation developed through selection in relation to resistance to herpes simplex virus 1 or 2 infections, which can be severe in newborn populations. ${ }^{54}$

\section{Summary}

There are numerous candidate genes under investigation for the development of cleft palate, each resulting in mutations with the potential to cause VPI through structural or anatomic anomalies of the palate. These include investigations showing cleft palate with FGFR1-associated Kallmann syndrome and forehead genes (FOXC2). The pathways involving these genes are not fully understood and may affect the functionality of the velopharyngeal port in multiple ways. This is clearly seen in 22q11.3 deletions with regards to adenoid hypoplasia, muscle hypotonia, platybasia, neuroanatomical changes, and upper airway asymmetry. The majority of causative candidate genes have been validates through the use of murine models, typically with transgenic and knockout lines. However, employing a translational approach with animal models has been difficult because cleft lip/palate displays significant genetic heterogeneity. Contributing socioeconomic factors further complicate potential analogies between animal and human models. Another level of difficulty is added when trying to focus on the specific methods by which these pathways affect speech production at the velopharyngeal port. As understanding progresses, there will be a natural evolution in the treatment of velopharyngeal 
dysfunction while attempting to achieve the long-term goal of prevention.

\section{References}

1 Kirschner RE, Baylis AL. Craniofacial: Head and Neck Surgery and Pediatric Plastic Surgery. 3rd ed. Philadelphia, PA: Elsevier Saunders; 2012

2 Gart MS, Gosain AK. Surgical management of velopharyngeal insufficiency. Clin Plast Surg 2014;41(2):253-270

3 Perry JL. Anatomy and physiology of the velopharyngeal mechanism. Semin Speech Lang 2011;32(2):83-92

4 Passavant G. Ueber die Beseitigung der naselnden. Sprache bei angeborenen Splaten des harten und weichen Gaumens (Gaumensegel-Schlundnacht und Ruccklagerung des Gaumensegels) [in German]. Arch Klin Chir 1865;2(37):333-349

5 Sloan GM. Posterior pharyngeal flap and sphincter pharyngoplasty: the state of the art. Cleft Palate Craniofac J 2000;37(2): 112-122

6 Padgett EC. The repair of cleft palates after unsuccessful operations, with special reference to cases with an extensive loss of palatal tissue. Arch Surg 1930;20:453-472

7 Hynes W. The results of pharyngoplasty by muscle transplantation in "failed cleft palate" cases, with special reference to the influence of the pharynx on voice production. 1953. Br J Plast Surg 1993; 46(5):430-439

8 Hynes W. Pharyngoplasty by muscle transplantation. Br J Plast Surg 1950;3(2):128-135

9 Orticochea M. Construction of a dynamic muscle sphincter in cleft palates. Plast Reconstr Surg 1968;41(4):323-327

10 Jackson IT, Silverton JS. The sphincter pharyngoplasty as a secondary procedure in cleft palates. Plast Reconstr Surg 1977;59(4): 518-524

11 Riski JE, Serafin D, Riefkohl R, Georgiade GS, Georgiade NG. A rationale for modifying the site of insertion of the orticochea pharyngoplasty. Plast Reconstr Surg 1984;73(6):882-894

12 Eckstein $\mathrm{H}$. Demonstration of parrafin prosthesis in defects of the face and palate. Dermatologica 1904;11:772-778

13 Blocksma R. Correction of velopharyngeal insufficiency by Silastic pharyngeal implant. Plast Reconstr Surg 1963;31:268274

14 Moon JB, Kuehn DP. Anatomy and Physiology of Normal and Disordered Velopharyngeal Function for Speech. 4th ed. Austin: Pro Ed; 1997

15 Kummer AW. Cleft Palate and Craniofacial Anomalies: Effects on Speech and Resonance. 2nd ed. Clifton Park, NY: Thomson Delmar Learning; 2008

16 Kosowski TR, Weathers WM, Wolfswinkel EM, Ridgway EB. Cleft palate. Semin Plast Surg 2012;26(4):164-169

17 Boorman JG, Sommerlad BC. Levator palati and palatal dimples: their anatomy, relationship and clinical significance. Br J Plast Surg 1985;38(3):326-332

18 Abe M, Murakami G, Noguchi M, Kitamura S, Shimada K, Kohama GI. Variations in the tensor veli palatini muscle with special reference to its origin and insertion. Cleft Palate Craniofac J 2004;41(5):474-484

19 Barsoumian R, Kuehn DP, Moon JB, Canady JW. An anatomic study of the tensor veli palatini and dilatator tubae muscles in relation to eustachian tube and velar function. Cleft Palate Craniofac J 1998; 35(2):101-110

20 Flores RL, Jones BL, Bernstein J, Karnell M, Canady J, Cutting CB. Tensor veli palatini preservation, transection, and transection with tensor tenopexy during cleft palate repair and its effects on eustachian tube function. Plast Reconstr Surg 2010;125(1): 282-289
21 Kuehn DP. Velopharyngeal anatomy and physiology. Ear Nose Throat J 1979;58(7):316-321

22 Moon JB, Smith AE, Folkins JW, Lemke JH, Gartlan M. Coordination of velopharyngeal muscle activity during positioning of the soft palate. Cleft Palate Craniofac J 1994;31(1):45-55

23 Fritzell B. The velopharyngeal muscles in speech. An electromyographic and cinéradiographic study. Acta Otolaryngol 1969(Suppl 250):250, 1

24 Kuehn DP, Azzam NA. Anatomical characteristics of palatoglossus and the anterior faucial pillar. Cleft Palate J 1978;15(4): 349-359

25 Huang MH, Lee ST, Rajendran K. A fresh cadaveric study of the paratubal muscles: implications for eustachian tube function in cleft palate. Plast Reconstr Surg 1997;100(4):833-842

26 Dickson DR, Dickson WM. Velopharyngeal anatomy. J Speech Hear Res 1972;15(2):372-381

27 Ruda JM, Krakovitz P, Rose AS. A review of the evaluation and management of velopharyngeal insufficiency in children. Otolaryngol Clin North Am 2012;45(3):653-669, viii

28 Perez E, Sullivan KE. Chromosome 22q11.2 deletion syndrome (DiGeorge and velocardiofacial syndromes). Curr Opin Pediatr 2002;14(6):678-683

29 Driscoll DA, Spinner NB, Budarf ML, et al. Deletions and microdeletions of 22q11.2 in velo-cardio-facial syndrome. Am J Med Genet 1992;44(2):261-268

30 Witt PD, Marsh JL, Marty-Grames L, Muntz HR, Gay WD. Management of the hypodynamic velopharynx. Cleft Palate Craniofac J 1995;32(3):179-187

31 Havkin N, Tatum SA, Shprintzen RJ. Velopharyngeal insufficiency and articulation impairment in velo-cardio-facial syndrome: the influence of adenoids on phonemic development. Int J Pediatr Otorhinolaryngol 2000;54(2-3):103-110

32 Ricchetti ET, States L, Hosalkar HS, et al. Radiographic study of the upper cervical spine in the 22q11.2 deletion syndrome. J Bone Joint Surg Am 2004;86-A(8):1751-1760

33 Chegar BE, Tatum SA III, Marrinan E, Shprintzen RJ. Upper airway asymmetry in velo-cardio-facial syndrome. Int J Pediatr Otorhinolaryngol 2006;70(8):1375-1381

34 Mitnick RJ, Bello JA, Shprintzen RJ. Brain anomalies in velo-cardiofacial syndrome. Am J Med Genet 1994;54(2):100-106

35 Wang PP, Solot C, Moss EM, et al. Developmental presentation of 22q11.2 deletion (DiGeorge/velocardiofacial syndrome). J Dev Behav Pediatr 1998;19(5):342-345

36 Papangeli I, Scambler P. The 22q11 deletion: DiGeorge and velocardiofacial syndromes and the role of TBX1. Wiley Interdiscip Rev Dev Biol 2013;2(3):393-403

37 Merscher S, Funke B, Epstein JA, et al. TBX1 is responsible for cardiovascular defects in velo-cardio-facial/DiGeorge syndrome. Cell 2001;104(4):619-629

38 Jerome LA, Papaioannou VE. DiGeorge syndrome phenotype in mice mutant for the T-box gene, Tbx1. Nat Genet 2001;27(3): 286-291

39 Zhang Z, Cerrato F, Xu H, et al. Tbx1 expression in pharyngeal epithelia is necessary for pharyngeal arch artery development. Development 2005;132(23):5307-5315 Erratum in: Development 2005;132(24):5614

40 Huynh T, Chen L, Terrell P, Baldini A. A fate map of Tbx1 expressing cells reveals heterogeneity in the second cardiac field. Genesis 2007;45(7):470-475

41 Kelly RG, Jerome-Majewska LA, Papaioannou VE. The del22q11.2 candidate gene $\mathrm{Tbx} 1$ regulates branchiomeric myogenesis. Hum Mol Genet 2004;13(22):2829-2840

42 Guo C, Sun Y, Zhou B, et al. A Tbx1-Six1/Eya1-Fgf8 genetic pathway controls mammalian cardiovascular and craniofacial morphogenesis. J Clin Invest 2011;121(4):1585-1595 Erratum in: J Clin Invest 2011;121(5):2060 
43 Choi M, Klingensmith J. Chordin is a modifier of tbx1 for the craniofacial malformations of 22q11 deletion syndrome phenotypes in mouse. PLoS Genet 2009;5(2):e1000395

44 Fulcoli FG, Huynh T, Scambler PJ, Baldini A. Tbx1 regulates the BMP-Smad1 pathway in a transcription independent manner. PLoS ONE 2009;4(6):e6049

45 Setó-Salvia N, Stanier P. Genetics of cleft lip and/or cleft palate: association with other common anomalies. Eur J Med Genet 2014; 57(8):381-393

46 Kondo S, Schutte BC, Richardson RJ, et al. Mutations in IRF6 cause Van der Woude and popliteal pterygium syndromes. Nat Genet 2002;32(2):285-289

47 Blanton SH, Cortez A, Stal S, Mulliken JB, Finnell RH, Hecht JT. Variation in IRF6 contributes to nonsyndromic cleft lip and palate. Am J Med Genet A 2005;137A(3):259-262

48 Satokata I, Maas R. Msx1 deficient mice exhibit cleft palate and abnormalities of craniofacial and tooth development. Nat Genet 1994;6(4):348-356

49 van den Boogaard MJ, Dorland M, Beemer FA, van Amstel HK. MSX1 mutation is associated with orofacial clefting and tooth agenesis in humans. Nat Genet 2000;24(4): 342-343

50 Zhang Z, Song Y, Zhao X, Zhang X, Fermin C, Chen Y. Rescue of cleft palate in Msx1-deficient mice by transgenic Bmp4 reveals a network of BMP and Shh signaling in the regulation of mammalian palatogenesis. Development 2002;129(17):4135-4146

51 Sözen MA, Suzuki K, Tolarova MM, Bustos T, Fernández Iglesias JE, Spritz RA. Mutation of PVRL1 is associated with sporadic, nonsyndromic cleft lip/palate in northern Venezuela. Nat Genet 2001; 29(2):141-142

52 Suzuki K, Hu D, Bustos T, et al. Mutations of PVRL1, encoding a cell-cell adhesion molecule/herpesvirus receptor, in cleft lip/ palate-ectodermal dysplasia. Nat Genet 2000;25(4):427-430

53 Geraghty RJ, Krummenacher C, Cohen GH, Eisenberg RJ, Spear PG. Entry of alphaherpesviruses mediated by poliovirus receptorrelated protein 1 and poliovirus receptor. Science 1998; 280(5369):1618-1620

54 Malik S, Wilcox ER, Naz S. Novel lip pit phenotypes and mutations of IRF6 in Van der Woude syndrome patients from Pakistan. Clin Genet 2014;85(5):487-491 